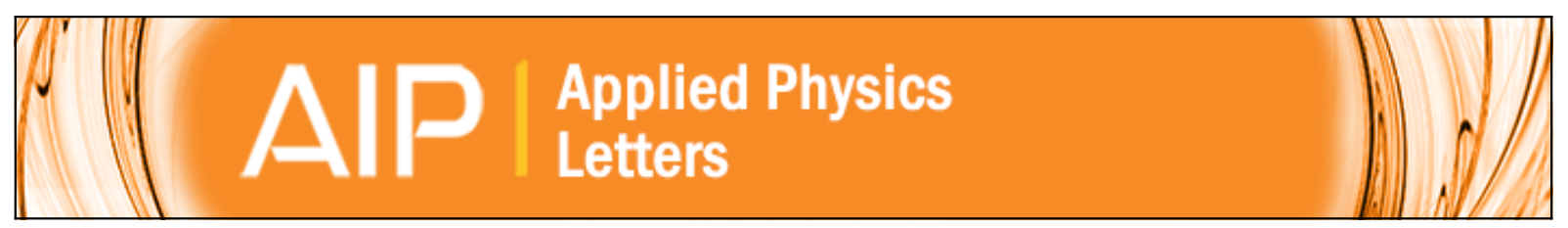

\title{
Optimal experimental design for the detection of light atoms from high-resolution scanning transmission electron microscopy images
}

J. Gonnissen, A. De Backer, A. J. den Dekker, G. T. Martinez, A. Rosenauer, J. Sijbers, and S. Van Aert

Citation: Applied Physics Letters 105, 063116 (2014); doi: 10.1063/1.4892884

View online: http://dx.doi.org/10.1063/1.4892884

View Table of Contents: http://scitation.aip.org/content/aip/journal/apl/105/6?ver=pdfcov

Published by the AIP Publishing

\section{Articles you may be interested in}

Combining nanocalorimetry and dynamic transmission electron microscopy for in situ characterization of materials processes under rapid heating and cooling

Rev. Sci. Instrum. 85, 084902 (2014); 10.1063/1.4892537

Compact design of a transmission electron microscope-scanning tunneling microscope holder with threedimensional coarse motion

Rev. Sci. Instrum. 74, 4945 (2003); 10.1063/1.1614872

High-speed transmission electron microscope

Rev. Sci. Instrum. 74, 4369 (2003); 10.1063/1.1611612

High-resolution x-ray diffraction and high-resolution scanning electron microscopy studies of Si-based structures with a buried amorphous layer

J. Appl. Phys. 84, 6076 (1998); 10.1063/1.368919

An instrument for electron beam and light transmission imaging of mass distribution in paper and fibrous webs

Rev. Sci. Instrum. 69, 2495 (1998); 10.1063/1.1148949

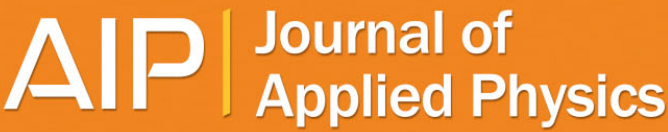

Journal of Applied Physics is pleased to announce André Anders as its new Editor-in-Chief 


\title{
Optimal experimental design for the detection of light atoms from high-resolution scanning transmission electron microscopy images
}

\author{
J. Gonnissen, ${ }^{1}$ A. De Backer, ${ }^{1}$ A. J. den Dekker, ${ }^{2,3}$ G. T. Martinez, ${ }^{1}$ A. Rosenauer, ${ }^{4}$ \\ J. Sijbers, ${ }^{2}$ and S. Van Aert ${ }^{1, a)}$ \\ ${ }^{1}$ Electron Microscopy for Materials Science, University of Antwerp, Groenenborgerlaan 171, 2020 Antwerp, \\ Belgium \\ ${ }^{2}$ iMinds-Vision Lab, University of Antwerp, Universiteitsplein 1, 2610 Wilrijk, Belgium \\ ${ }^{3}$ Delft Center for Systems and Control, Delft University of Technology, Mekelweg 2, 2628 CD Delft, \\ The Netherlands \\ ${ }^{4}$ Institute of Solid State Physics, University of Bremen, Otto-Hahn-Allee NW1, 28359 Bremen, Germany
}

(Received 7 April 2014; accepted 30 July 2014; published online 13 August 2014)

\begin{abstract}
We report an innovative method to explore the optimal experimental settings to detect light atoms from scanning transmission electron microscopy (STEM) images. Since light elements play a key role in many technologically important materials, such as lithium-battery devices or hydrogen storage applications, much effort has been made to optimize the STEM technique in order to detect light elements. Therefore, classical performance criteria, such as contrast or signal-to-noise ratio, are often discussed hereby aiming at improvements of the direct visual interpretability. However, when images are interpreted quantitatively, one needs an alternative criterion, which we derive based on statistical detection theory. Using realistic simulations of technologically important materials, we demonstrate the benefits of the proposed method and compare the results with existing approaches. (C) 2014 AIP Publishing LLC. [http://dx.doi.org/10.1063/1.4892884]
\end{abstract}

The importance of enhancing the imaging power to detect light atoms, such as lithium and hydrogen, and to visualize mono-atomic-layer membranes, such as graphene, has re-attracted interest in the optimization of the scanning transmission electron microscopy (STEM) detector for such applications. Findlay et al. have shown that so-called annular bright field (ABF) STEM, whereby an annular detector is used with the detector collection range lying within the cone of illumination (the direct-scattered region), performs well to detect light elements. ${ }^{1,2}$ Another strength of ABF STEM is that both light and heavy atom columns are visible simultaneously, in contrast to high angle annular dark field (HAADF) STEM, which tends to render light elements invisible when in proximity to heavier scatterers. ${ }^{3,4}$ This effect can be understood since the ADF contrast, also called Zcontrast, is based on the scattering amplitude, while the ABF phase-contrast is based on wave-interference, so that it only requires the atoms to alter the phase of the electron wave. ${ }^{5}$ As direct imaging of light elements is necessary for the full determination of crystal structures, such as cathode materials for lithium-ion batteries, this topic has recently become very important and a lot of research has been done in this field in the past few years. Not only the identification of individual lithium atoms ${ }^{6-9}$ but also the direct imaging of other light elements, such as carbon, oxygen or nitrogen, ${ }^{2,10-12}$ and even hydrogen ${ }^{13}$ has been investigated. In these studies, the experimental settings are often determined in terms of direct visual interpretability using classical performance measures, such as contrast or signal-to-noise ratio (SNR). However, if images are interpreted quantitatively, classical performance measures are no longer appropriate. ${ }^{14-21} \mathrm{We}$ will therefore

a)Sandra.VanAert@uantwerpen.be provide a theoretical tool to quantitatively obtain the optimal annular detector settings to detect individual light atoms using the principles of detection theory. ${ }^{22}$ The concept of this theory has been introduced in Ref. 14 showing preliminary results when considering the simplest STEM imaging model of an isolated atom. Here, both the theory will significantly be extended and realistic image simulations to accurately describe experimental images will be used ${ }^{23-26}$ allowing us to demonstrate our method to technologically important materials.

When considering the problem of quantitatively identifying the atomic number $Z$ from a STEM image, a priori knowledge about the atom types that may be present in the sample and their concentration ratios is usually available. In such cases, the question reduces to distinguishing between a finite plausible set of values for the atomic numbers $Z$, given the experimental STEM observations. In this paper, we will restrict ourselves to the problem of deciding between two hypotheses, where (a) each hypothesis corresponds to the assumption of a specific $Z$ value, or (b) where the alternative hypothesis describes the absence of an atom of any type. The hypotheses for both situations are then summarized as follows:

$$
\begin{aligned}
& \text { (a) } \mathcal{H}_{0}: \mathrm{Z}=\mathrm{Z}_{0} \quad \text { (b) } \mathcal{H}_{0}: \mathrm{Z}=\mathrm{Z}_{0} \\
& \mathcal{H}_{1}: \mathrm{Z}=\mathrm{Z}_{1} \quad \mathcal{H}_{1}: \mathrm{Z} \in \emptyset \text {, }
\end{aligned}
$$

where $\mathcal{H}_{0}$ denotes the null hypothesis and $\mathcal{H}_{1}$ the alternative hypothesis. Note, however that detection theory also provides the tools to generalize this problem to choose between more than two hypotheses. ${ }^{22}$ Throughout this paper, we will assume that a priori knowledge assures that only $\mathcal{H}_{0}$ or $\mathcal{H}_{1}$ is possible, so that one of both hypotheses is always correct. Furthermore, prior probabilities $P\left(\mathcal{H}_{0}\right)$ and $P\left(\mathcal{H}_{1}\right)$, with 
$P\left(\mathcal{H}_{0}\right)+P\left(\mathcal{H}_{1}\right)=1$, are assumed to be known. In this way, we express a prior belief in the likelihood of the hypotheses. If both hypotheses are equally likely, then it is reasonable to assign equal prior probabilities of $1 / 2$. In a quantitative approach, the goal is now to minimize the probability of assigning the wrong hypothesis. In a so-called Bayesian approach, this probability of error $\mathrm{P}_{\mathrm{e}}$ is defined as

$$
\mathrm{P}_{\mathrm{e}}=P\left(\mathcal{H}_{0} \mid \mathcal{H}_{1}\right) P\left(\mathcal{H}_{1}\right)+P\left(\mathcal{H}_{1} \mid \mathcal{H}_{0}\right) P\left(\mathcal{H}_{0}\right)
$$

where $P\left(\mathcal{H}_{i} \mid \mathcal{H}_{j}\right)$ is the conditional probability of deciding $\mathcal{H}_{i}$ when $\mathcal{H}_{j}$ is true. Using criterion (1), the two possible errors are weighted appropriately to yield an overall error measure. Decision rules are now defined such that the probability of error is minimized. For this purpose, it is shown ${ }^{22}$ that we should then decide $\mathcal{H}_{1}$ if

$$
\frac{p_{\mathbf{w}}\left(\mathbf{w} ; \mathcal{H}_{1}\right)}{p_{\mathbf{w}}\left(\mathbf{w} ; \mathcal{H}_{0}\right)}>\frac{P\left(\mathcal{H}_{0}\right)}{P\left(\mathcal{H}_{1}\right)}=\gamma
$$

with $p_{\mathbf{w}}\left(\mathbf{w} ; \mathcal{H}_{i}\right)$ the conditional joint probability function (PF) $p_{\mathbf{w}}\left(\omega ; \mathcal{H}_{i}\right)$ assuming $\mathcal{H}_{i}$ to be true, evaluated at the available observations $\mathbf{w}$. When assuming that the STEM observations are statistically independent electron counting results, which are modeled as a Poisson distribution, this joint $\mathrm{PF}$ is given by

$$
p_{\mathbf{w}}\left(\omega ; \mathcal{H}_{i}\right)=\prod_{n=1}^{N} \prod_{m=1}^{M} \frac{\lambda_{n m}^{\omega_{n m}}}{\omega_{n m} !} \exp \left(-\lambda_{n m}\right)
$$

where the parameter $\lambda_{n m}$ corresponds to the expectation values for the observations, $\mathbb{E}\left[\mathbf{w}_{n m}\right]$, and the index $n m$ corresponds to the probe at the position $\left(x_{n}, y_{m}\right)^{T}$ for a set of $N M$ observations. Since these expectations will depend on which hypothesis $\mathcal{H}_{i}$ is assumed to be true, also the PF depends on $\mathcal{H}_{i}$. In general, these expectations can be computed using software that allows one to simulate STEM images for a given input material's structure and a given set of microscope parameters. $^{27}$ If the prior probabilities are assumed to be equal such that $\gamma$ in Eq. (2) corresponds to 1, we decide $\mathcal{H}_{1}$ if

$$
\ln L R(\mathbf{w}) \equiv \ln \left(\frac{p_{\mathbf{w}}\left(\mathbf{w} ; \mathcal{H}_{1}\right)}{p_{\mathbf{w}}\left(\mathbf{w} ; \mathcal{H}_{0}\right)}\right)>\ln (1)=0
$$

otherwise $\mathcal{H}_{0}$ is decided. This corresponds to choosing the hypothesis for which the log-likelihood function is maximal. The function $L R(\mathbf{w})$ is called the likelihood ratio since it indicates for each set of observations $\mathbf{w}$ the likelihood of $\mathcal{H}_{1}$ versus the likelihood of $\mathcal{H}_{0}$. The left-hand side of Eq. (4) is termed the log-likelihood ratio. Given decision rule Eq. (4), the probability of error $\mathrm{P}_{\mathrm{e}}$, given by Eq. (1), can be reformulated as follows:

$$
\mathrm{P}_{\mathrm{e}}=\frac{1}{2} P\left(\ln L R(\mathbf{w})<0 \mid \mathcal{H}_{1}\right)+\frac{1}{2} P\left(\ln L R(\mathbf{w})>0 \mid \mathcal{H}_{0}\right) .
$$

When using the joint PF given by Eq. (3), the log-likelihood ratio can be rewritten as

$$
\ln L R(\mathbf{w})=\sum_{n=1}^{N} \sum_{m=1}^{M} \mathbf{w}_{n m} \ln \frac{\lambda_{n m, \mathcal{H}_{1}}}{\lambda_{n m, \mathcal{H}_{0}}}-\lambda_{n m, \mathcal{H}_{1}}+\lambda_{n m, \mathcal{H}_{0}} .
$$

Following the central limit theorem, the log-likelihood ratio tends to be normally distributed. The expected value $\mu$ and variance $\sigma^{2}$, characterizing this normal distribution, can be computed from Eq. (6) when assuming $\mathcal{H}_{i}$ to be true, giving the following results:

$$
\begin{gathered}
\mu_{\mathcal{H}_{i}}=\sum_{n=1}^{N} \sum_{m=1}^{M} \lambda_{n m, \mathcal{H}_{i}} \ln \frac{\lambda_{n m, \mathcal{H}_{1}}}{\lambda_{n m, \mathcal{H}_{0}}}-\lambda_{n m, \mathcal{H}_{1}}+\lambda_{n m, \mathcal{H}_{0}} \\
\sigma_{\mathcal{H}_{i}}^{2}=\sum_{n=1}^{N} \sum_{m=1}^{M} \lambda_{n m, \mathcal{H}_{i}}\left(\ln \frac{\lambda_{n m, \mathcal{H}_{1}}}{\lambda_{n m, \mathcal{H}_{0}}}\right)^{2}
\end{gathered}
$$

In this derivation, use is made of the property that the variance of a Poisson distributed observation equals its expectation value. The explicit description of the distribution of the log-likelihood ratio now enables us to unambiguously compute the probability of error given by Eq. (5)

$$
\mathrm{P}_{\mathrm{e}}=\frac{1}{2}\left[\Phi\left(\frac{-\mu_{\mathcal{H}_{1}}}{\sigma_{\mathcal{H}_{1}}}\right)+\Phi\left(\frac{\mu_{\mathcal{H}_{0}}}{\sigma_{\mathcal{H}_{0}}}\right)\right]
$$

with $\Phi(x)$ the cumulative distribution function of the standard normal distribution. Expression (9) greatly facilitates the computation of the probability to assign the wrong hypothesis. As compared to alternatives suggested in Ref. 14, the current approach no longer involves the analysis of many repetitive noise realisations or the use of the so-called Kullback-Leibler divergence, which is a measure for experimental design based on the distance between the distributions of the log-likelihood ratio under the two hypotheses, without taking overlap into account. In contrast, the probability of error $\mathrm{P}_{\mathrm{e}}$ can now be calculated in a straightforward manner and can be used as an alternative quantitative criterion to optimize the STEM detector settings in terms of choosing the right hypothesis.

As a first application to show the reliability of our method, we investigate the crystal $\mathrm{YH}_{2}$ which is referred to as one of the most thermodynamically stable hydrides. ${ }^{13}$ One has been able to detect $\mathrm{H}$ in this material using ABF STEM detector settings, ${ }^{13}$ and it is our goal to find out if we obtain the same optimal detector type, or even better the exact optimal detector angles, using our quantitative approach. For this case, the two hypotheses $\mathcal{H}_{0}$ and $\mathcal{H}_{1}$ now correspond to the presence of $\mathrm{H}$ atoms and the absence of atoms of any type, respectively. For this problem, we have simulated expectation models for a $2.6 \mathrm{~nm}$ thick $\mathrm{YH}_{2}$ crystal in the presence and absence of the hydrogen atoms using the STEMsim software under the multislice approach. ${ }^{27}$ These expectation models are simulated under Scherzer conditions for a probe forming aperture angle of $21.8 \mathrm{mrad}$, an electron dose of $2000 e^{-} / \AA^{2}$ and an acceleration voltage of $300 \mathrm{kV}$. Source size broadening is taken into account by convoluting point source images with a Gaussian with FWHM $=0.7 \AA$. Next, the probability of error is calculated using Eq. (9). In Fig. 1, the distributions of the log-likelihood ratios are shown for three different detector settings (11-53 mrad, 22-53 $\operatorname{mrad}=\mathrm{ADF}$, and $11-17 \mathrm{mrad}=\mathrm{ABF})$. The distributions of the log-likelihood ratios in red and green correspond to the presence or absence of a $\mathrm{H}$ column, respectively. The overlap between the distributions of both hypotheses corresponds 

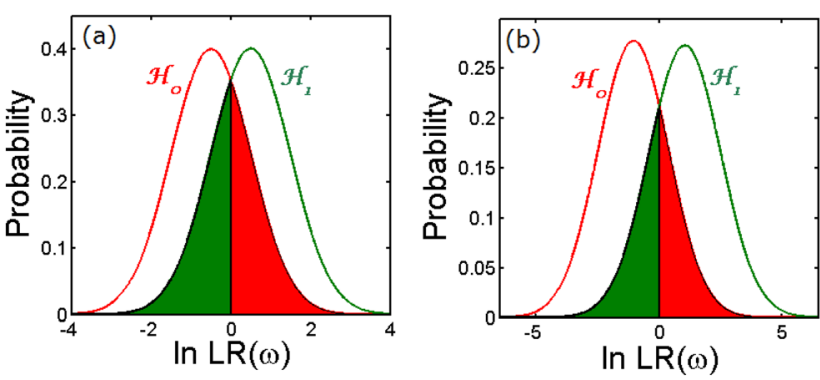

to the probability of error. By calculating the probability of error for a broad range of inner and outer detector radii, it can be investigated which settings minimize $\mathrm{P}_{e}$. The results are shown in Fig. 2(a). From this figure, it can be found that $\mathrm{P}_{e}$ is smallest for a detector collection range of 11-17 mrad, corresponding to the ABF STEM region. For the ABF detector setting, the distributions of the log-likelihood ratios are more separated, as can be observed from the comparison of the different example results presented in Fig. 1. Note also that local optima are found for the conventional ADF STEM setting (for a detector collection range, starting from an inner detector angle of $22 \mathrm{mrad}$ ), although this is not what we would expect from qualitative contrast measures. Simulated STEM images of $\mathrm{YH}_{2}$ are shown in Fig. 3 for the three different detector settings. Note that the scale is different for the three detector settings since the amount of signal changes with the detection area. Using this quantitative method, not only a suggested optimal annular detector type is found (e.g., $\mathrm{ABF}$ or $\mathrm{ADF}$ ) but also the optimal angles for the detection range are derived in an objective, quantitative way.

As a second application to demonstrate our method, we look at the interface of a $\mathrm{SrTiO}_{3} / \mathrm{LaAlO}_{3}$ substrate, which is

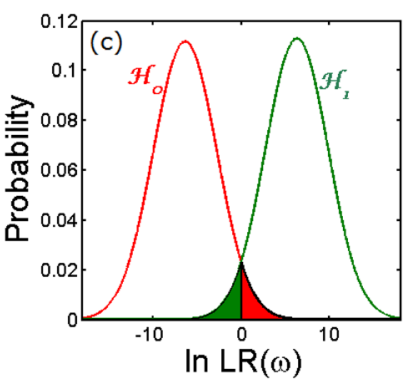

FIG. 1. Log-likelihood ratio distributions for detector collection angles of (a) 11-53 mrad, (b) 22-53 mrad, and (c) 11-17 mrad in case of the presence (red) and absence (green) of $\mathrm{H}$ in $\mathrm{YH}_{2}$. The red and green colored areas correspond to the probability of deciding $\mathcal{H}_{0}$ while $\mathcal{H}_{1}$ is true and the probability of deciding $\mathcal{H}_{1}$ while $\mathcal{H}_{0}$ is true, respectively.

an interesting case study since such interfaces are known to exhibit a plethora of exceptional properties. ${ }^{28}$ If we want to decide whether there is a $\mathrm{Ti}$ or $\mathrm{Al}$ atom at the interface, we can describe this problem as a binary hypothesis test with hypotheses: $\mathcal{H}_{0}: \mathrm{Z}=\mathrm{Al}$ and $\mathcal{H}_{1}: \mathrm{Z}=\mathrm{Ti}$. Similar as before, we can optimize the detector settings in order to minimize the probability to choose the wrong hypothesis. A $1.6 \mathrm{~nm}$ crystal is simulated with either $\mathrm{Al}$ or $\mathrm{Ti}$ atoms at the interface. An example is shown in Fig. 4 for an annular detector collection range of 23-100 mrad. Using Eq. (9), $\mathrm{P}_{e}$ can again be calculated as a function of the inner and outer radius of the annular STEM detector. The result of the probability of error is given in Fig. 2(b). Based on this figure, we find that the suggested optimal detector setting lies in the low angle annular dark field (LAADF) regime, with the inner detector angle just larger than the probe forming angle (from 23 to $100 \mathrm{mrad})$. Hovden and Muller ${ }^{10}$ also found that for wellresolved and atomically thin specimens, the LAADF detector can provide a significant increase in SNR over other common detector geometries including $\mathrm{ABF}$ and incoherent $\mathrm{BF}$.

As a third example to demonstrate our method, we look at the case where we want to detect a lithium atom in the
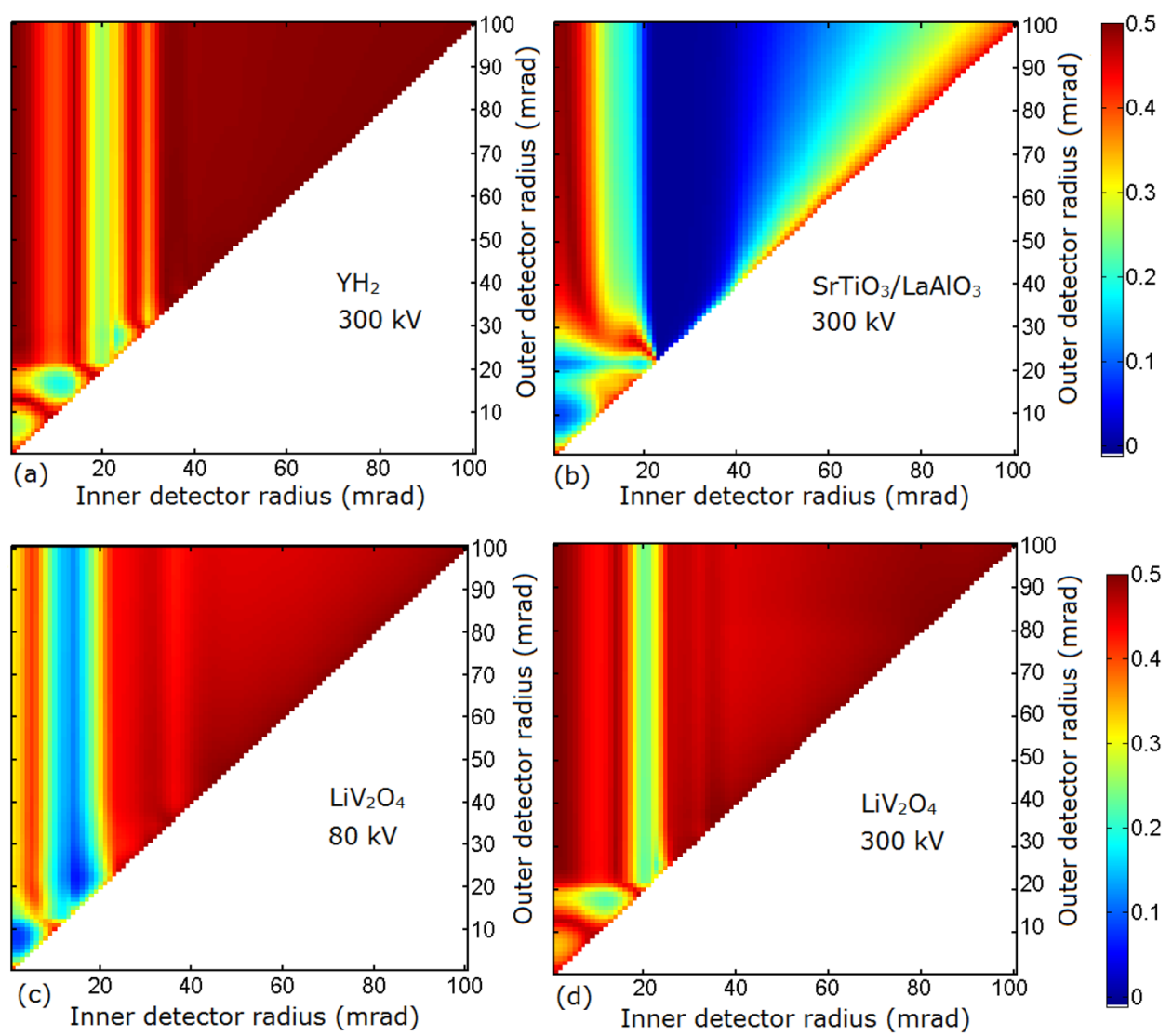

FIG. 2. $\mathrm{P}_{e}$ as a function of the inner and outer detector angle at Scherzer conditions for an electron dose of 2000 $e^{-} / \AA^{2}$ and a probe forming angle of $21.8 \mathrm{mrad}$ for (a) $\mathrm{YH}_{2}$ at $300 \mathrm{kV}$, (b) $\mathrm{SrTiO}_{3} / \mathrm{LaAlO}_{3}$ at $300 \mathrm{kV}$, (c) $\mathrm{LiV}_{2} \mathrm{O}_{4}$ at $80 \mathrm{kV}$, and (d) $\mathrm{LiV}_{2} \mathrm{O}_{4}$ at $300 \mathrm{kV}$. 


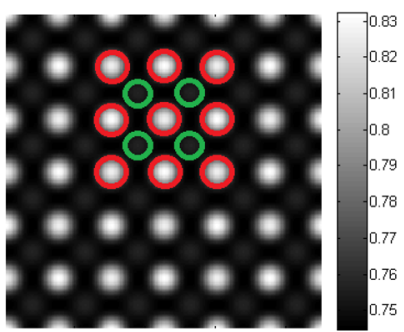

(a)

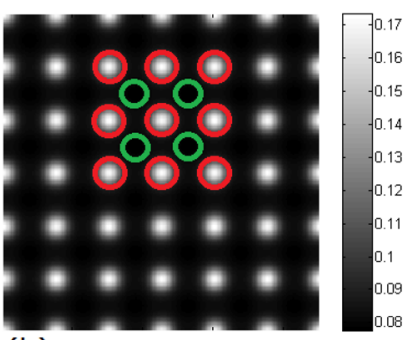

(b)

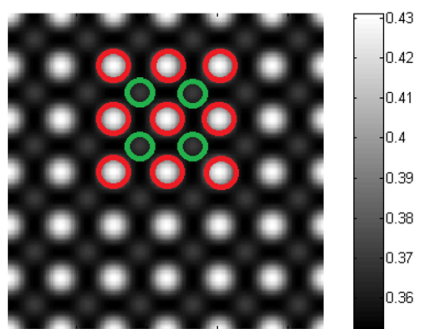

(c)

FIG. 3. Simulated STEM images of $\mathrm{YH}_{2}$ viewed from the [010] direction, for annular detector collection ranges of (a) 11-53 mrad, (b) 22-53 mrad, and (c) 11-17 mrad.

(a)
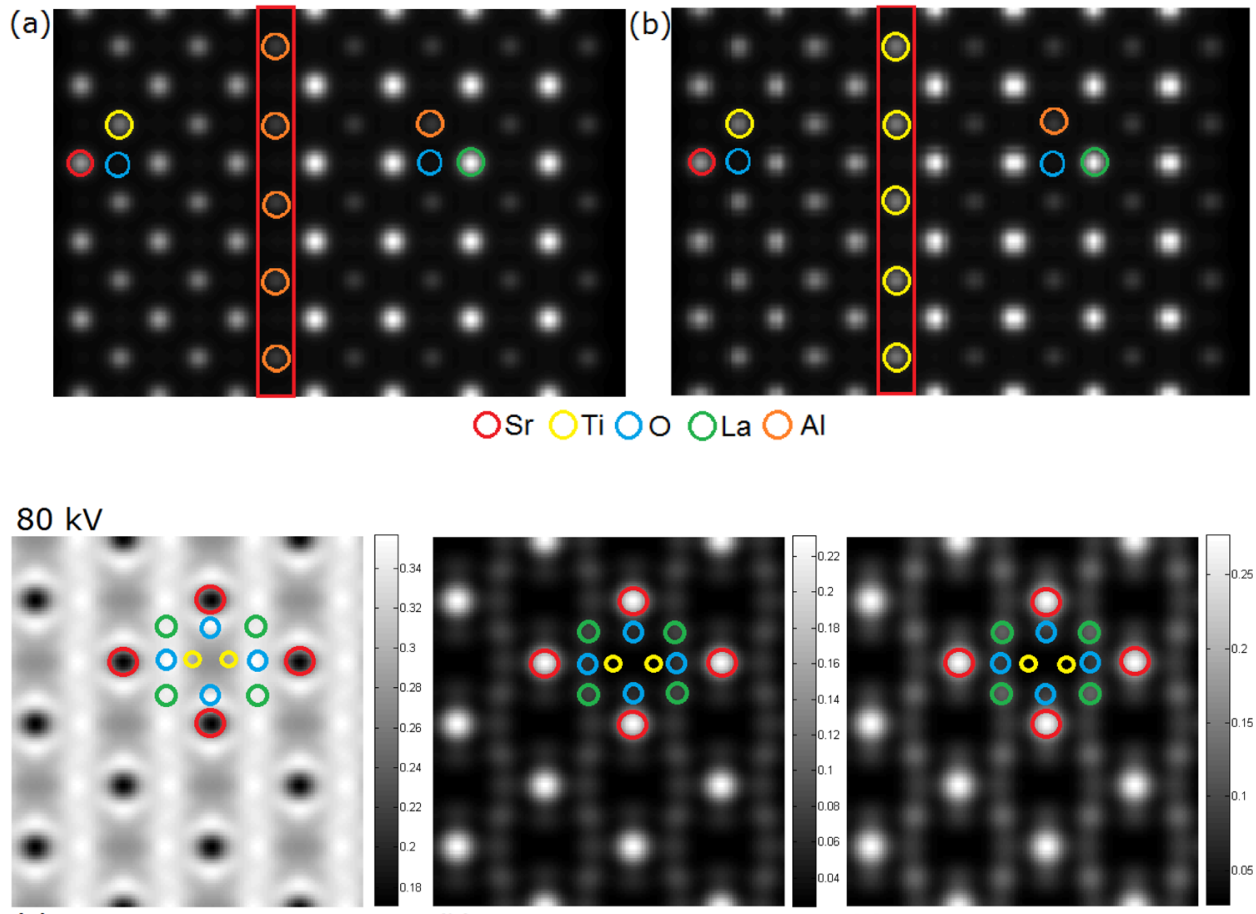

(a) BF

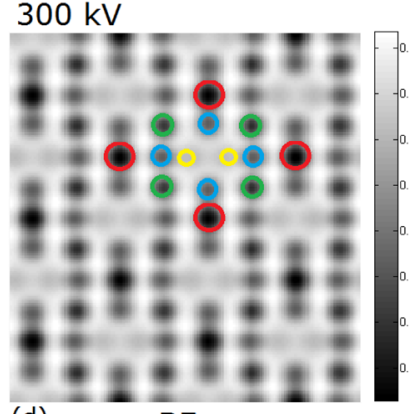

(d) $\mathrm{BF}$

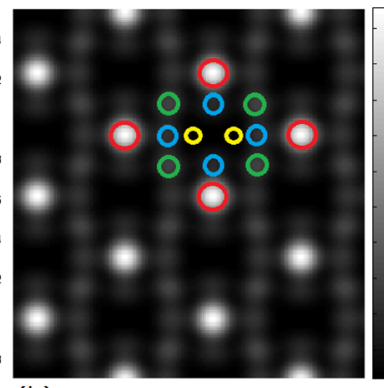

ABF

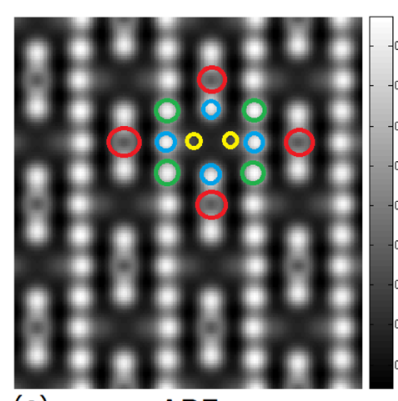

(e)

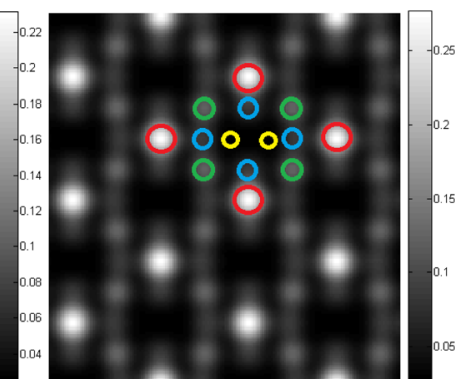

(c)

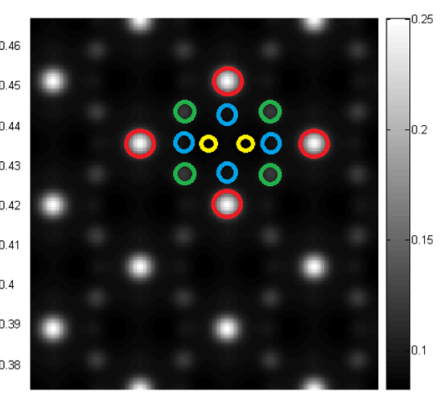

(f)

ADF
FIG. 4. Simulated STEM images of the $\mathrm{SrTiO}_{3} / \mathrm{LaAlO}_{3}$ compound with (a) $\mathrm{Al}$ atoms or (b) $\mathrm{Ti}$ atoms at the interface for the optimal detector collection range of 23-100 mrad. crystal $\mathrm{LiV}_{2} \mathrm{O}_{4}$ from a STEM image. For this material, we consider the problem of optimizing the STEM detector in order to detect a lithium column. In Ref. 7, individual lithium columns could be visualized using ABF STEM for an acceleration voltage of $300 \mathrm{kV}$ and a sample thickness of $2.9 \mathrm{~nm}$. In order to compare with these results, we optimized detector settings for $300 \mathrm{kV}$. In addition, since one could prefer to perform this experiment at a lower voltage in order to reduce beam damage, we also optimized the design for $80 \mathrm{kV}$. The expectation models are thus simulated for a $2.9 \mathrm{~nm}$ crystal in the presence and absence of lithium, for both accelerating voltages. Simulations at $80 \mathrm{kV}$ are also performed for a probe forming aperture angle of $21.8 \mathrm{mrad}$ assuming Scherzer conditions for the spherical aberration and defocus. Simulated
STEM images of $\mathrm{LiV}_{2} \mathrm{O}_{4}$ are shown in Fig. 5 for three different detector settings at $80 \mathrm{kV}$ (upper row) and $300 \mathrm{kV}$ (lower row). Using the same procedure as in our previous examples, $\mathrm{P}_{e}$ can be computed as a function of the detector settings. The results for both accelerating voltages are shown in Figs. 2(c) and 2(d). For the acceleration voltage of $300 \mathrm{kV}$, the $\mathrm{ABF}$ STEM regime is found as an optimum when following our quantitative approach with a detector ranging from 12 to 18 mrad. Local optima are also found in the ADF STEM regime, similarly to the $\mathrm{YH}_{2}$ example. For the lower accelerating voltage of $80 \mathrm{kV}$ a slightly different result is found. Here, we obtain an optimum for a detector collection range of 15-21 mrad, which also lies in the ABF STEM regime, but there are no local optima found in the ADF STEM regime. The 
optimum in the BF STEM regime on the other hand becomes more pronounced and the probability of error reaches almost an equally small value for a detector ranging from 2 to $8 \mathrm{mrad}$ as for 15 to $21 \mathrm{mrad}$. It is interesting to note that for the same incident electron dose, light atoms become easier to detect at $80 \mathrm{kV}$ than at $300 \mathrm{kV}$ (as can be observed from the lower values for the probability of error), although at the expense of direct visual interpretability of the light atoms. As a function of crystal thickness, it can be shown that the ABF STEM regime remains optimum to detect lithium.

In this work, we proposed a method based on detection theory to find the optimal detector settings for different kinds of applications. In our approach, the probability of error $\mathrm{P}_{e}$ is introduced as a quantitative measure which can replace classical criteria such as contrast or SNR. It is interesting to note that based on simulation studies, the ABF STEM detector setting comes out as the optimal annular detector setting to detect light atoms in the proximity of heavier scatterers, as suggested elsewhere. ${ }^{2,6-8,11,13} \mathrm{We}$ also noticed local optima for the conventional ADF setting when using an accelerating voltage of $300 \mathrm{kV}$, which are not expected based on contrast comparisons. Also for a lower accelerating voltage of $80 \mathrm{kV}$, the ABF STEM setting was found to be optimal in order to detect lithium in the $\mathrm{LiV}_{2} \mathrm{O}_{4}$ crystal. In the case of deciding between the presence of two different atom types at an interface of a thin crystal, the LAADF STEM detector setting is proposed as optimal annular detector setting, as suggested elsewhere. ${ }^{10}$ Not only the optimal detector type is found using this quantitative approach but also the optimal detector angles follow from the proposed method. The methodology is demonstrated using realistic simulations and may help designing the next generation of STEM detectors, where a greater flexibility in the choice of detector areas is provided. ${ }^{29,30}$

In conclusion, the proposed method can be applied to a wide range of materials applications and will provide objective suggestions for the inner and outer angle of the annular STEM detector in order to detect specific atoms with the lowest probability of error. Obviously, the present analysis can easily be extended to include other microscope settings including convergence angle, defocus, and spherical aberration.

The authors acknowledge financial support from the Research Foundation Flanders (FWO, Belgium) through project fundings (G.0393.11, G.0064.10, and G.0374.13) and a Ph.D. Grant to A. De Backer. The research leading to these results has received funding from the European Union Seventh Framework Programme [FP7/2007-2013] under Grant Agreement No. 312483 (ESTEEM2) and from the Deutsche Forschungsgemeinschaft under Contract No. RO2057/8-1.
${ }^{1}$ S. D. Findlay, N. Shibata, H. Sawada, E. Okunishi, Y. Kondo, T. Yamamoto, and Y. Ikuhara, Appl. Phys. Lett. 95, 191913 (2009).

${ }^{2}$ S. D. Findlay, N. Shibata, H. Sawada, E. Okunishi, Y. Kondo, and Y. Ikuhara, Ultramicroscopy 110, 903-923 (2010).

${ }^{3}$ M. Varela, A. Lupini, K. Van Benthem, A. Borisevich, M. Chisholm, N. Shibata, E. Abe, and S. J. Pennycook, Annu. Rev. Mater. Res. 35, 539 (2005).

${ }^{4}$ K. Mkhoyan, P. Baston, J. Cha, W. Schaff, and J. Silcox, Science 312, 1354 (2006).

${ }^{5}$ J. M. Cowley, Appl. Phys. Lett. 15, 58-59 (1969).

${ }^{6}$ S. D. Findlay, N. R. Lugg, N. Shibata, L. J. Allen, and Y. Ikuhara, Ultramicroscopy 111, 1144-1154 (2011).

${ }^{7}$ S. Lee, Y. Oshima, H. Sawada, F. Hosokawa, and E. Okunishi, J. Appl. Phys. 109, 113530 (2011).

${ }^{8}$ Y. Oshima, H. Sawada, F. Hosokawa, E. Okunishi, T. Kaneyama, Y. Kondo, S. Niitaka, H. Takagi, Y. Tanishiro, and K. Takayanagi, J. Electron Microsc. 59, 457-461 (2010).

${ }^{9}$ R. Huang and Y. Ikuhara, Curr. Opin. Solid State Mater. Sci. 16, 31-38 (2012).

${ }^{10}$ R. Hovden and D. A. Muller, Ultramicroscopy 123, 59-65 (2012).

${ }^{11}$ E. Okunishi, I. Ishikawa, H. Sawada, F. Hosokawa, M. Hori, and Y. Kondo, Microsc. Microanal. 15, 164-165 (2009).

${ }^{12}$ E. Okunishi, H. Sawada, and Y. Kondo, Micron 43, 538-544 (2012).

${ }^{13}$ R. Ishikawa, E. Okunishi, H. Sawada, Y. Kondo, F. Hosokawa, and E. Abe, Nat. Mater. 10, 278-281 (2011).

${ }^{14}$ A. J. den Dekker, J. Gonnissen, A. De Backer, J. Sijbers, and S. Van Aert, Ultramicroscopy 134, 34-43 (2013).

${ }^{15}$ D. Van Dyck, S. Van Aert, A. J. den Dekker, and A. van den Bos, Ultramicroscopy 98, 27-42 (2003).

${ }^{16}$ A. J. den Dekker, S. Van Aert, D. Van Dyck, and A. van den Bos, Ultramicroscopy 104, 83-106 (2005).

${ }^{17}$ A. J. den Dekker, S. Van Aert, D. Van Dyck, A. van den Bos, and P. Geuens, Ultramicroscopy 89, 275-290 (2001).

${ }^{18}$ S. Van Aert, A. J. den Dekker, D. Van Dyck, and A. van den Bos, J. Struct. Biol. 138, 21-33 (2002).

${ }^{19}$ S. Van Aert, A. J. den Dekker, and D. Van Dyck, Micron 35, 425-429 (2004).

${ }^{20}$ S. Van Aert, A. J. den Dekker, D. Van Dyck, and A. van den Bos, Ultramicroscopy 90, 273-289 (2002).

${ }^{21}$ S. Van Aert, D. Van Dyck, and A. J. den Dekker, Opt. Express 14, 3830-3839 (2006).

${ }^{22}$ S. M. Kay, Fundamentals of Statistical Signal Processing. Volume II Detection Theory (Prentice-Hall, Inc., New Jersey, 2009).

${ }^{23}$ J. M. LeBeau, S. D. Findlay, L. J. Allen, and S. Stemmer, Phys. Rev. Lett. 100, 206101 (2008).

${ }^{24}$ S. Van Aert, A. De Backer, G. T. Martinez, B. Goris, S. Bals, G. Van Tendeloo, and A. Rosenauer, Phys. Rev. B 87, 064107 (2013).

${ }^{25}$ A. Rosenauer, K. Gries, K. Mueller, A. Pretorious, M. Schowalter, A. Avramescu, K. Engl, and S. Lutgen, Ultramicroscopy 109, 1171-1182 (2009).

${ }^{26}$ T. Grieb, K. Mueller, R. Fritz, M. Schowalter, N. Neugebohrn, N. Knaub, K. Volz, and A. Rosenauer, Ultramicroscopy 117, 15-23 (2012).

${ }^{27}$ A. Rosenauer and M. Schowalter, Springer Proc. Phys. 120, 169-172 (2007).

${ }^{28}$ M. Huijben, G. Rijnders, D. H. A. Blank, S. Bals, S. Van Aert, J. Verbeeck, G. Van Tendeloo, A. Brinkman, and H. Hilgenkamp, Nat. Mater. 5, 556-560 (2006).

${ }^{29}$ N. Shibata, Y. Kohno, S. D. Findlay, H. Sawada, Y. Kondo, and Y. Ikuhara, J. Electron Microsc. 59, 473-479 (2010).

${ }^{30}$ M. Lohr, R. Schregle, M. Jetter, C. Wächter, T. Wunderer, F. Scholz, and J. Zweck, Ultramicroscopy 117, 7-14 (2012). 